\title{
Neutron production in terrestrial gamma ray flashes
}

\author{
B. E. Carlson, ${ }^{1}$ N. G. Lehtinen, ${ }^{1}$ and U. S. Inan ${ }^{1,2}$ \\ Received 28 July 2009; revised 10 November 2009; accepted 9 December 2009; published 21 April 2010.
}

[1] Terrestrial gamma ray flashes (TGFs) are brief bursts of photons with energies up to $20 \mathrm{MeV}$ typically observed in association with lightning. Such energetic photons may undergo photonuclear reactions with nontrivial cross section in the vicinity of the giant dipole resonance. Pulses of neutrons have been observed experimentally in coincidence with lightning, suggesting such reactions are observable. We present simulations of expected photoneutron production based on initial conditions inferred from observations of TGFs. We predict an average of $\sim 10^{12}$ neutrons produced per TGF and give energy, time, and space distributions of neutrons produced and neutrons reaching ground and satellite altitude. The simulation results are consistent with some observations and suggest further experiments may be profitable.

Citation: Carlson, B. E., N. G. Lehtinen, and U. S. Inan (2010), Neutron production in terrestrial gamma ray flashes, J. Geophys. Res., 115, A00E19, doi:10.1029/2009JA014696.

\section{Introduction}

[2] Terrestrial gamma ray flashes (TGFs) are intense pulses of energetic photons observed by satellites with a fluence of $\sim 1$ photon $/ \mathrm{cm}^{2}$, a time scale of $\sim 0.5 \mathrm{~ms}$ and photon energies of up to $20 \mathrm{MeV}$ when seen from orbit [Fishman et al., 1994; Smith et al., 2005; Grefenstette et al., 2008, 2009]. TGFs are typically observed in close time coincidence with detectable lightning discharge within $300 \mathrm{~km}$ of the subsatellite point [Inan et al., 2006; Cohen et al., 2006; Cummer et al., 2005; Stanley et al., 2006]. Though the details of the production mechanism are the subject of much discussion with many mechanisms proposed [see Inan and Lehtinen, 2005; Lehtinen et al., 1996; Milikh and Valdivia, 1999; Fargion, 2002; Paiva et al., 2009; Milikh et al., 2005; Dwyer, 2008; Carlson et al., 2009], the rough properties of TGFs are known. In particular the very hard average spectrum of observed TGF photons requires a bremsstrahlung photon source altitude of 15-20 km [Dwyer and Smith, 2005; Carlson et al., 2007]. Assuming the photons are uniformly distributed over a disk of radius $300 \mathrm{~km}$ (given by the typical lightning-subsatellite distance), the 1 photon $/ \mathrm{cm}^{2}$ fluence implies that of order $10^{15}$ photons reach satellite altitude. Full comparison of satellite observations to simulations of photon attenuation and scattering in the atmosphere requires a source of photons with $15-20 \mathrm{~km}$ altitude, uniform photon directional distribution with zenith angle $\theta<45^{\circ}$, and a total source of $4 \times 10^{15}$ to $4 \times 10^{16}$ photons on average, with broader beams requiring source populations as high as $10^{17}$ photons [Carlson et al., 2007].

[3] This large number of photons with an energy spectrum extending up to and above $20 \mathrm{MeV}$ suggests that

\footnotetext{
${ }^{1}$ Space, Telecommunications and Radioscience Laboratory, Electrical Engineering Department, Stanford University, Stanford, California, USA.

${ }^{2}$ Koç University, Istanbul, Turkey.

Copyright 2010 by the American Geophysical Union. 0148-0227/10/2009JA014696
}

photonuclear reactions should occur. For example, the dominant process for nitrogen, photoneutron production, ${ }^{14} \mathrm{~N}(\gamma, n){ }^{13} \mathrm{~N}$, occurs for photons with energies $\mathcal{E}_{\gamma}>$ $10.55 \mathrm{MeV}$ with an energy-dependent cross section exhibiting a large peak at $\sim 23 \mathrm{MeV}$, the so-called giant dipole resonance [Chadwick et al., 2000; Berman and Fultz, 1975]. For nitrogen, the peak cross section is $\sim 14$ millibarns. For comparison, the pair production and Compton scattering cross sections for photons in this energy range are roughly equal and are approximately 170 millibarns [Cullen et al., 1997]. Photonuclear processes therefore amount to a maximum of about $5 \%$ of the total photon interaction cross section. This implies Compton scattering and pair production are dominant and that neutron production does not significantly affect energetic photon behavior. Energetic neutrons, however, if they are produced in large enough quantities, are interesting as they are highly penetrating and are not easily attenuated. For context, the mean free path for energetic photons at sea level is of order $1 \mathrm{~km}$ (pair production and Compton scattering dominate), while the mean free path for energetic neutrons is of order $0.3 \mathrm{~km}$ (elastic scattering dominates). TGFs should therefore be accompanied by neutron production.

[4] This idea has been suggested previously in work by Leonid Babich and others. Specifically, Babich [2006] raises the possibility of photonuclear reactions associated with TGFs in the context of the upward atmospheric discharge model described by Babich et al. [2004]. Babich et al. [2007] and Babich et al. [2008] further develop this idea, and Babich and Roussel-Dupré [2007] describe the relation of these analyses to the existing experiments discussed below. Overall, these works indicate that the upward atmospheric discharge model produces $\sim 10^{15}$ neutrons, but the properties of these neutrons are not specified and the results of the upward atmospheric discharge model may be questionable in the context of TGFs given the extremely poor spectral fits seen in Figure 5 of Babich et al. [2008]. 
[5] Neutron bursts have been reported in conjunction with lightning indicating $10^{9}-10^{10}$ neutrons are produced per lightning discharge [Shah et al., 1985; Shyam and Kaushik, 1999]. Neutron count rates observed from orbit show occasional excess count rates in equatorial regions suggested to be caused by neutron production by lightning [BratolyubovaTsulukidze et al., 2004]. Whether neutrons observed in conjunction with lightning are produced by photonuclear processes in a TGF-like event is uncertain. Fusion processes have also been suggested as relevant [Libby and Lukens, 1973], but more detailed analysis indicates photonuclear processes are more likely [Babich, 2006].

[6] In this paper, we use the inferred source spectrum of terrestrial gamma ray flashes to drive Monte Carlo simulations of photoneutron production. The simulation results are used to predict the properties of the neutrons produced and the properties of neutrons that may be observable. Our results indicate that TGF production at $15-20 \mathrm{~km}$ altitude should be accompanied by production of $3 \times 10^{11}$ to $3 \times 10^{12}$ neutrons. This is fewer than the predictions of $10^{15}$ neutrons produced by Babich's upward atmospheric discharge model, but is more than that suggested to be produced in conjunction with lightning. We additionally give the energy, arrival time, and position distributions of neutrons as produced and at ground and satellite altitudes. Our results are consistent with the ground observations of lightning-associated neutron pulses from Shah et al. [1985] and Shyam and Kaushik [1999] but are not consistent with satellite observations of BratolyubovaTsulukidze et al. [2004].

\section{Simulation of Neutron Production by TGFs}

[7] We predict neutron production by Monte Carlo simulations of photonuclear reactions caused by TGF photons. We use the GEANT4 software package [Agostinelli et al., 2003], a high-energy physics Monte Carlo simulation tool that includes all relevant electromagnetic and nuclear physics including Compton scattering, pair production, photoneutron production, neutron elastic and inelastic scatter, and radiative capture. Photonuclear reactions for nitrogen, oxygen, and argon, in particular, are approximated in the giant dipole resonance regime with a parametrization and interpolation scheme involving data from ${ }^{12} \mathrm{C},{ }^{16} \mathrm{O},{ }^{27} \mathrm{Al}$ and ${ }^{40} \mathrm{Ca}$. Comparison of the cross section used in GEANT4 to measured cross sections shows that this scheme matches the cross section to better than a factor of 2 , with systematic underestimate of the cross section just above the minimum energy threshold [Kossov, 2002].

[8] The initial conditions for these simulations are chosen to match our knowledge of TGFs and to cover reasonable scenarios for neutron emission. First consider a population of electrons with energy distribution characteristic of electricfield-driven avalanche growth of populations of relativistic particles as described by Lehtinen et al. [1999]. The bremsstrahlung spectrum produced by these electrons does not depend strongly on the strength of the electric field or the altitude of the electric field region, provided the electric field exceeds the runaway relativistic electron avalanche threshold [Dwyer and Smith, 2005]. We start our simulation with photons drawn from this spectrum, ignoring photons with energies below the lowest relevant photoneutron production threshold (9.87 MeV for argon). Only approximately $1 \%$ of the original TGF source photons exceed this cutoff energy. While there are undoubtedly spectral differences between TGFs, this initial photon spectrum matches the observed average TGF spectrum very well for source altitudes 15$20 \mathrm{~km}$ [Carlson et al., 2007] and thus provides a good measure of the average TGF initial photon spectrum.

[9] These initial photons are placed in point sources at 2.5, $5,7.5,10,15$, and $20 \mathrm{~km}$ altitudes, spanning the allowed range of altitudes for TGF production and extending to lower altitudes to explore possible ground observations. Such point sources are useful as they produce the most compact source of neutrons and can be superposed to construct larger distributed sources. The atmospheric density profile is taken from Hedin [1991]. At each altitude, the initial photon directions are either distributed isotropically or are distributed uniformly over a cone with half-opening angle $20^{\circ}$ directed either upward or downward. Photons may be emitted isotropically by electrons driven by nontrivial electric field structure, while $20^{\circ}$ beams are characteristic of bremsstrahlung from runaway relativistic electron avalanches driven by uniform electric fields. The behavior of these initial photons is then simulated with GEANT4. Every neutron produced in the simulation is tracked and neutrons that travel below $0.5 \mathrm{~km}$ altitude (ground) or above $350 \mathrm{~km}$ altitude (representing escape to satellite altitude) are recorded. One million initial photons are simulated for each set of initial conditions. The numbers of neutrons produced and recorded in these simulations are given in Table 1.

[10] As discussed previously, $4 \times 10^{15}$ to $4 \times 10^{16}$ energetic photons are produced in an average TGF as observed by RHESSI [Carlson et al., 2007]. Of these source photons, an average of $\sim 1 \%$ or $\sim 10^{14}$ exceed the threshold for photonuclear reactions. The numbers in Table 1 give approximately 6400 neutrons produced per million photons with energy above the photonuclear reaction cutoff, giving $3 \times 10^{11}$ to $3 \times$ $10^{12}$ neutrons produced in an average TGF. This is roughly two orders of magnitude lower than the $10^{14}-10^{15}$ previously predicted [Babich et al., 2007, 2008; Babich, 2006], a fact we return to in section 3 .

[11] In the initial simulations no neutrons were recorded for certain conditions, for example neutrons that reach ground for upward directed initial photons at $20 \mathrm{~km}$. This does not mean neutron observation under such conditions is impossible, only that it is not resolved in our initial simulations. To improve the statistics, we run a second round of simulations, starting not with energetic photons, but with energetic neutrons as produced in our first round of simulations. This improves the statistics of neutron propagation but does not improve the statistics of neutron production. The improved estimates of the number of neutrons matching the given conditions from this second round of simulations are given in parentheses in Table 1.

[12] As the initial photon energy distribution is constant and photoneutron production takes place almost entirely with unscattered photons, the produced neutron energy distribution is roughly constant and is shown as the solid black line in Figure 1. The mean produced neutron energy is $3.9 \mathrm{MeV}$ (median $3.6 \mathrm{MeV}$ ). Figure 1 also shows sample energy spectra of neutrons reaching ground and satellite levels (black dotted and gray solid lines, respectively). Such neutrons have been partially thermalized after propagation through the atmosphere and have a mean energy 
Table 1. Neutrons Produced and Observed in Simulations per Million Initial Energetic Photons ${ }^{\mathrm{a}}$

\begin{tabular}{|c|c|c|c|c|}
\hline \multirow[b]{2}{*}{ Initial Altitude $(\mathrm{km})$} & \multirow[b]{2}{*}{ Record Type } & \multicolumn{3}{|c|}{ Initial Direction Distribution } \\
\hline & & Up & Down & Isotropic \\
\hline \multirow[t]{3}{*}{2.5} & prod. & 6468 & 6343 & 6436 \\
\hline & esc. & $<1\left(<5 \times 10^{-3}\right)$ & $<1\left(<5 \times 10^{-3}\right)$ & $<1\left(<5 \times 10^{-3}\right)$ \\
\hline & gnd. & $1(0.32)$ & $229(237)$ & $23(26.6)$ \\
\hline \multirow[t]{3}{*}{5} & prod. & 6425 & 6485 & 6427 \\
\hline & esc. & $<1(0.13)$ & $<1\left(<5 \times 10^{-3}\right)$ & $<1\left(<5 \times 10^{-3}\right)$ \\
\hline & gnd. & $<1\left(<5 \times 10^{-3}\right)$ & $6(6.5)$ & $<1(0.04)$ \\
\hline \multirow{3}{*}{7.5} & prod. & 6434 & 6433 & 6343 \\
\hline & esc. & $4(4.7)$ & $<1\left(<5 \times 10^{-3}\right)$ & $2(0.32)$ \\
\hline & gnd. & $<1\left(<5 \times 10^{-3}\right)$ & $<1(0.28)$ & $<1\left(<5 \times 10^{-3}\right)$ \\
\hline \multirow[t]{3}{*}{10} & prod. & 6475 & 6420 & 6337 \\
\hline & esc. & $34(38.0)$ & $<1(0.03)$ & $3(4.5)$ \\
\hline & gnd. & $<1\left(<5 \times 10^{-3}\right)$ & $<1(0.04)$ & $<1\left(<5 \times 10^{-3}\right)$ \\
\hline \multirow[t]{3}{*}{15} & prod. & 5756 & 6323 & 6221 \\
\hline & esc. & $452(500)$ & $15(13.9)$ & $113(142)$ \\
\hline & gnd. & $<1\left(<5 \times 10^{-3}\right)$ & $<1\left(<5 \times 10^{-3}\right)$ & $<1\left(<5 \times 10^{-3}\right)$ \\
\hline \multirow[t]{3}{*}{20} & prod. & 4250 & 6349 & 5923 \\
\hline & esc. & $1107(1238)$ & $205(228)$ & $684(794)$ \\
\hline & gnd. & $<1\left(<5 \times 10^{-3}\right)$ & $<1\left(<5 \times 10^{-3}\right)$ & $<1\left(<5 \times 10^{-3}\right)$ \\
\hline
\end{tabular}

${ }^{a}$ Numbers are for simulations of one million initial photons with a typical TGF energy spectrum but with energy $\mathcal{E}_{\gamma}>9.87 \mathrm{MeV}$, with $<1$ indicating no neutrons were observed. Parenthetical numbers give the number of neutrons observed per million initial photons if the neutrons produced in the photon simulations are reproduced 200 times and allowed to propagate in the atmosphere to improve the statistics of propagation.

of $2.0 \mathrm{MeV}$ (median $1.3 \mathrm{MeV}$ ) with a substantially shifted distribution.

[13] Sample distributions of neutron production positions are shown in Figure 2. The neutrons are often produced far from the initial photon source, consistent with the energetic photon mean free path of $\sim 1 \mathrm{~km} \times \exp (z / H)$ where $z$ is the altitude and $H$ is the atmospheric scale height $(H \sim 8 \mathrm{~km})$.

[14] The arrival time distributions for neutrons at ground and satellite altitudes from instantaneous photon point sources are shown in Figure 3. Neutrons arriving at ground level from point photon sources at $2.5 \mathrm{~km}$ and $5 \mathrm{~km}$ are shown in Figure 3 (left), while the time distribution for neutrons arriving at satellite altitudes from $15 \mathrm{~km}$ and $20 \mathrm{~km}$ are shown in Figure 3 (right). Ground level neutrons from an instantaneous photon source arrive in a pulse $\sim 10 \mu$ s long with a pronounced tail, a time short compared to a typical TGF time scale of $0.5 \mathrm{~ms}$. Neutrons arriving at satellite altitude are substantially more dispersed due to their longer path lengths, and arrive spread over a pulse $\sim 50 \mathrm{~ms}$ long, much longer than a typical TGF time scale. Consequently, ground level neutron arrival times should reflect the time scale of their production, while arrival times at satellite altitude are widely spread due to longer path lengths. Note also that the ground level neutron detections of Shah et al. [1985] show neutron arrival up to 10-100 ms after the trigger time, much longer than the typical arrival time delay shown in Figure 3. This delay suggests a source not intimately connected with the triggering electromagnetic signal. Such a time delay may be due to activity elsewhere in the discharge not directly associated with the process that resulted in the trigger.

[15] We also give results of simulations of isotropic point sources of neutrons drawn from the characteristic energy spectrum shown in Figure 1. Expected numbers of neutrons are shown in Table 2. These results are not representative of the distributed sources of neutrons shown in Figure 2, but are useful for understanding of photoneutron propagation in the atmosphere. In particular, a simple treatment of exponential attenuation and $r^{-2}$ decrease underestimates the flux by a factor of 6 for the first row in Table 2, with progressively worse error as altitude increases. Proper correction of this error by accounting for scattering effectively requires table lookup of accumulation factors, precisely the information provided in Table 2.

[16] Sample radial distributions of the flux of neutrons arriving at ground and satellite altitudes from point sources at various altitudes are shown in Figure 4. Neutrons at ground level cover a disk roughly $1 \mathrm{~km}$ in radius, while longer path lengths lead to a much broader $\sim 400 \mathrm{~km}$ radius disk at $350 \mathrm{~km}$ altitude. At ground level, higher source altitudes result in slightly broader neutron distribution, but the bulk of the neutrons still arrive less than $1 \mathrm{~km}$ from the subsource point if the source is less than $3 \mathrm{~km}$ from the ground.

\section{Discussion}

[17] On the basis of TGF observations and the resulting constraints on the source described by Carlson et al. [2007],

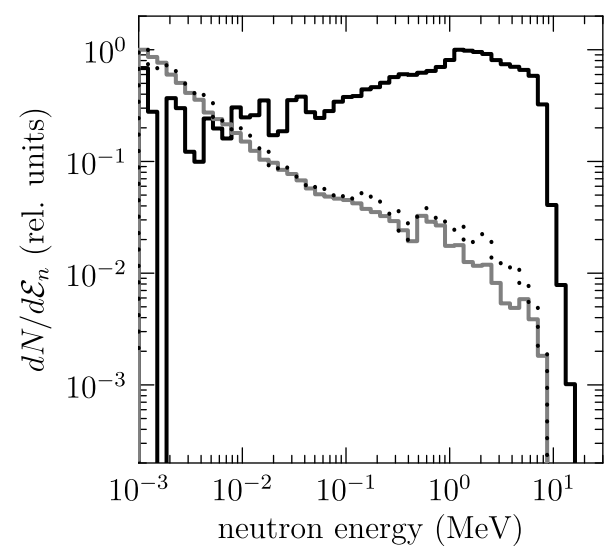

Figure 1. Characteristic energy spectrum of neutrons when produced (solid black curve). Also shown are the spectrum of neutrons reaching ground from a downward directed source at $2.5 \mathrm{~km}$ (black dotted) and satellite altitude from an upward directed source at $20 \mathrm{~km}$ (gray solid). 


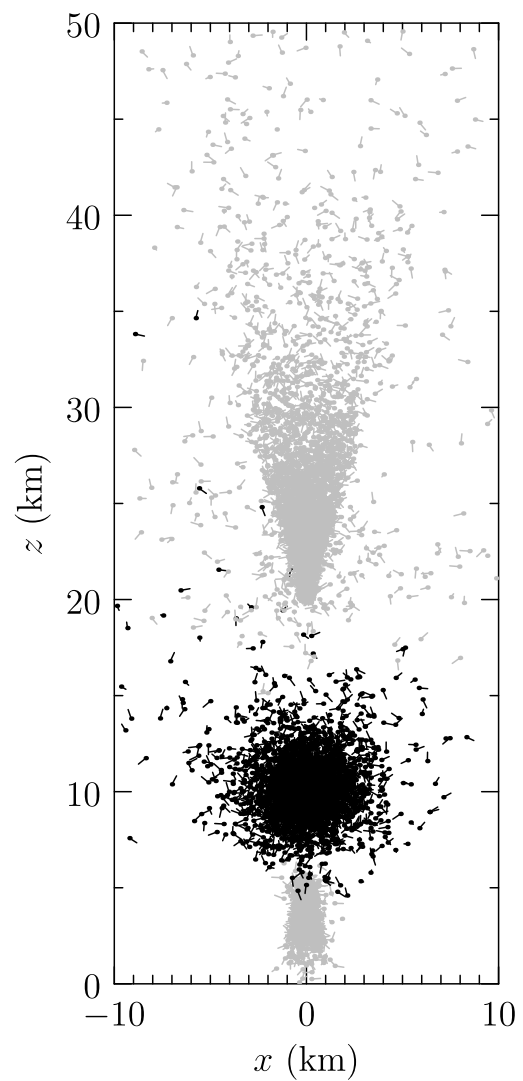

Figure 2. Position and direction of motion of neutrons when produced. Each dot represents the production a neutron, with the line segment extending outward in the direction of motion of the neutron. Top gray cluster, $20 \mathrm{~km}$ initial altitude, upward directed $20^{\circ}$ beam. Black cluster, $10 \mathrm{~km}$ initial altitude, isotropic. Bottom gray cluster, $5 \mathrm{~km}$ initial altitude, downward directed $20^{\circ}$ beam.

we estimate $\sim 10^{12}$ neutrons produced per TGF on average. This is a factor of $\sim 100$ smaller than existing predictions derived from a discharge model [Babich et al., 2007, 2008; Babich, 2006]. The discrepancy is likely due to different initial photon population sizes and different photon spectra. The discharge model of Babich [2006] predicts the TGF source photon population size to be $10^{1 f}$ with a spectrum capable of producing $10^{15}$ neutrons [Babich and RousselDupré, 2007], while the results described above together with those of Carlson et al. [2007] show that the RHESSI and BATSE TGF data require a source of $\sim 10^{16}$ photons with a spectrum shown here to be only capable of producing $10^{12}$ neutrons. Energetic processes lower in the atmosphere not associated with TGFs such as the gamma ray burst observed at ground level in association with triggered lightning described by Dwyer et al. [2004] may of course have different characteristics and correspondingly higher or lower photon and neutron yields. Babich and Roussel-Dupré [2007], for instance, estimates the photon yield for the Dwyer et al. [2004] event to be $3 \times 10^{19}$, though this is almost certainly an overestimate, with the resulting neutron yields given as $4 \times 10^{13}$.

[18] Assuming our estimate of $\sim 10^{12}$ neutrons produced in an average TGF, the number of neutrons arriving at ground level from a downward directed photon source at $5 \mathrm{~km}$ can be calculated from the numbers in Table 1 as $\sim 1 \times 10^{9}$. These neutrons, spread over a disk $1 \mathrm{~km}$ in radius give an average fluence of $3 \times 10^{-2} \mathrm{~cm}^{-2}$. A similar source at $2.5 \mathrm{~km}$ gives an average fluence of $1 \mathrm{~cm}^{-2}$. These fluxes are grossly consistent with the observations of Shah et al. [1985] which see pulses of more than one neutron with an effective area of $3 \times 10^{4} \mathrm{~cm}^{2}$ in temporal coincidence with lightning. Similar results were also observed by Shyam and Kaushik [1999], but they do not give the effective area of their detector so a full comparison is not possible. Both Shyam and Kaushik [1999] and Shah et al. [1985] assume the neutrons were produced locally by a lightning discharge and thus estimate lower numbers of neutrons produced. Our results here suggest that their results may also be explained by more distant neutron sources due to photonuclear processes. Note that as energetic photons have a longer mean free path than neutrons, photoproduction of neutrons implies that neutron observations should be accompanied by energetic photon observations.

[19] Neutrons that escape to $350 \mathrm{~km}$ altitude are spread over a region roughly $400 \mathrm{~km}$ in radius, giving a fluence of $10^{-5} \mathrm{~cm}^{-2}$ for the upward directed photon source at $15 \mathrm{~km}$

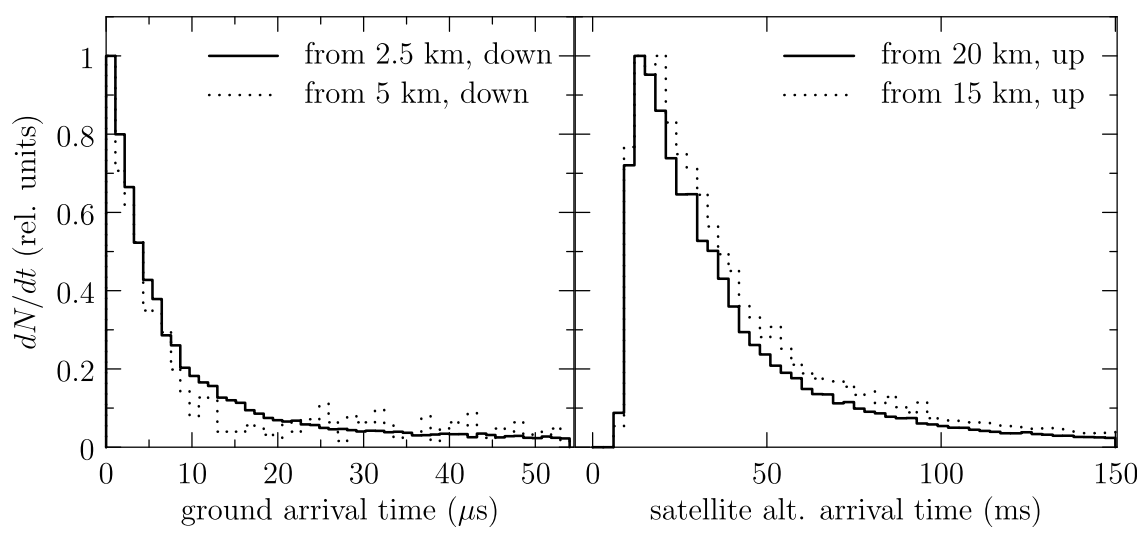

Figure 3. Neutron arrival time distributions. (left) Neutrons arriving at ground level from a downward directed photon source at $2.5 \mathrm{~km}$ altitude. (right) Neutrons arriving at $350 \mathrm{~km}$ altitude from an upward directed photon source at $20 \mathrm{~km}$ altitude. Note the different time scales. The long time scale in Figure 3 (right) is due to longer scattering path lengths, not merely longer path lengths from source to satellite plane. 
Table 2. Number of Neutrons That Reach Ground and Satellite Altitude per Neutron Injected in an Isotropic Point Source at the Indicated Altitude With the Characteristic Energy Spectrum Shown in Figure 1

\begin{tabular}{lcc}
\hline Altitude $(\mathrm{km})$ & Ground & Escape to Satellite \\
\hline 0.75 & 0.232 & $<3 \times 10^{-6}$ \\
1 & 0.0916 & $<3 \times 10^{-6}$ \\
1.25 & 0.0328 & $<3 \times 10^{-6}$ \\
1.5 & 0.0116 & $<3 \times 10^{-6}$ \\
1.75 & $4.2 \times 10^{-3}$ & $<3 \times 10^{-6}$ \\
2 & $1.4 \times 10^{-3}$ & $<3 \times 10^{-6}$ \\
2.25 & $4.2 \times 10^{-4}$ & $<3 \times 10^{-6}$ \\
2.5 & $1.7 \times 10^{-4}$ & $<3 \times 10^{-6}$ \\
2.75 & $7 \times 10^{-5}$ & $<3 \times 10^{-6}$ \\
3 & $1.6 \times 10^{-5}$ & $<3 \times 10^{-6}$ \\
3.25 & $1.1 \times 10^{-5}$ & $<3 \times 10^{-6}$ \\
3.5 & $5 \times 10^{-6}$ & $<3 \times 10^{-6}$ \\
5 & $<3 \times 10^{-6}$ & $<3 \times 10^{-6}$ \\
7 & $<3 \times 10^{-6}$ & $<3 \times 10^{-6}$ \\
10 & $<3 \times 10^{-6}$ & $<3 \times 10^{-6}$ \\
12 & $<3 \times 10^{-6}$ & $5.0 \times 10^{-4}$ \\
15 & $<3 \times 10^{-6}$ & $7.2 \times 10^{-4}$ \\
17 & $<3 \times 10^{-6}$ & 0.0396 \\
20 & $<3 \times 10^{-6}$ & 0.111 \\
25 & $<3 \times 10^{-6}$ & 0.318 \\
\hline
\end{tabular}

consistent with satellite observations of TGFs. Though the number of neutrons that escape to satellite altitude from highaltitude photon sources are greater than the numbers that reach ground level from low-altitude sources, the greater area covered by the emissions drastically reduces the flux. Bratolyubova-Tsulukidze et al. [2004] examined data from neutron detectors on satellites of effective area $30 \mathrm{~cm}^{2}$ and $100 \mathrm{~cm}^{2}$. With these effective areas, our results indicate that individual neutron bursts of the sort suggested here on the basis of TGF observations would be undetectable by the instruments analyzed. Statistically, optimistically assuming that every lightning discharge produces a TGF-like energetic photon burst and that every lightning discharge happens within $400 \mathrm{~km}$ of the satellite, the global lightning frequency of $40 \mathrm{~Hz}$ would give the count rate due to lightning-induced photonuclear processes as $4 \times 10^{-2} \mathrm{~Hz}$. As even this extremely optimistic estimate is below the lowest background count rate observed by Bratolyubova-Tsulukidze et al. [2004] of $\sim 0.5 \mathrm{~Hz}$, our results indicate that the observations of
Bratolyubova-Tsulukidze et al. [2004] are not due to photonuclear reactions in TGF-like processes.

[20] Photoneutrons produced by energetic photons in a TGF-like event should therefore be observable at ground level if the photon source has $\leq 5 \mathrm{~km}$ altitude. Our predictions are consistent with existing observations of neutron bursts associated with lightning. This suggests that these observations may be explained by photonuclear interactions of highenergy photons produced in a TGF-like process. The presence of photoneutrons indicates the presence of $\geq 10 \mathrm{MeV}$ photons, a strong proxy for the presence of energetic electrons and runaway relativistic electron avalanche, a process suggested as relevant to many aspects of thunderstorm and atmospheric physics including lightning initiation [e.g., Dwyer, 2005; Marshall et al., 2005; Gurevich et al., 1999], highfrequency radio emissions [e.g., Tierney et al., 2005], and sprite and blue jet formation [e.g., Lehtinen et al., 1999; Babich et al., 2007; Roussel-Dupré and Gurevich, 1996]. Further searches for these neutrons, including more detailed studies of their time and space distribution in relation to causative lightning, should therefore help shed light on these topics.

\section{Summary}

[21] We give the results of simulations of photoneutron production as motivated by TGF observations. Our results indicate that $\sim 10^{12}$ energetic neutrons are produced per TGF with distributions in energy, space and time as shown in Figures 1-4. The fractional production of photoneutrons and the fraction that reach ground level or escape to satellite altitude are given in Tables 1 and 2. If the photon source is at a sufficiently low altitude, these neutrons will be observable on the ground with temporal and radial distributions given in Figures 3 and 4 . The properties of the emissions predicted are consistent with ground observations of neutrons coincident with lightning discharge. Further observations of neutrons are necessary to clarify the connections between lightning, TGFs, neutrons, and other energetic atmospheric phenomena. In particular, measurements of the time and space distributions of lightning-associated neutron emissions could be compared to our results here and used to infer the properties of the neutron source. Such analysis would help answer such open questions as whether or not all lightning produces energetic

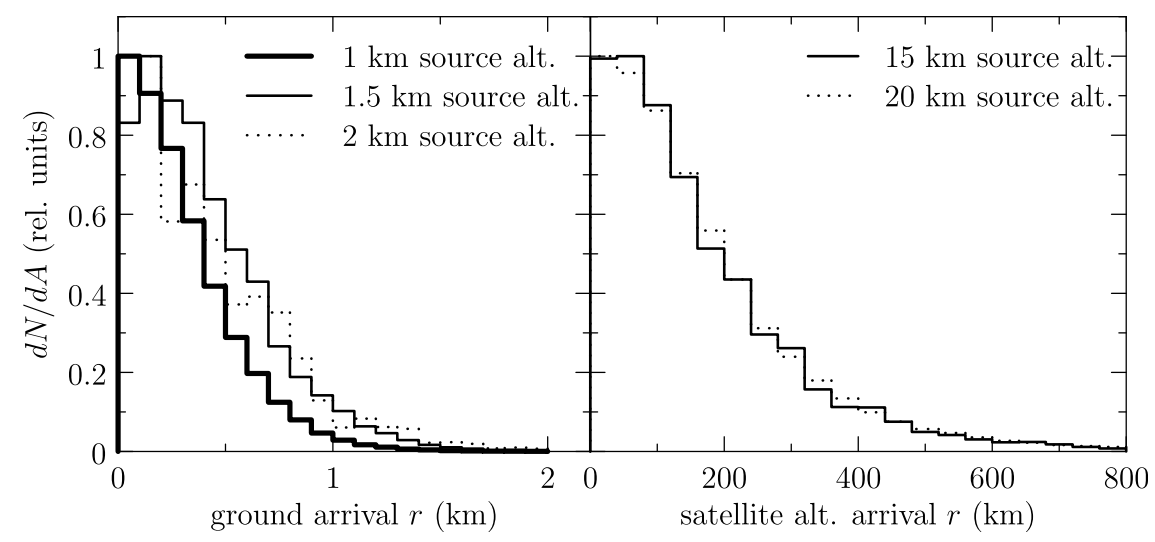

Figure 4. Number of neutrons per unit area versus radial distance from the subsource point for neutrons produced in a point source at the altitude indicated on the plot. (left) Ground level and (right) $350 \mathrm{~km}$ altitude. 
photons, whether or not all lightning produces energetic neutrons, and if coincidences between lightning and neutron measurements are always accompanied by energetic photons.

[22] Acknowledgments. The authors wish to thank Martin Walt for very useful discussions. This work was supported by the National Science Foundation under grant ATM-0535461.

[23] Amitava Bhattacharjee thanks the reviewers for their assistance in evaluating this paper.

\section{References}

Agostinelli, S., et al. (2003), G4-A simulation toolkit, Nucl. Instrum. Methods Phys. Res. Sect. A, 506(3), 250-303.

Babich, L. P. (2006), Generation of neutrons in giant upward atmospheric discharges, JETP Lett., 84, 285-288, doi:10.1134/S0021364006180020.

Babich, L. P., and R. A. Roussel-Dupré (2007), Origin of neutron flux increases observed in correlation with lightning, J. Geophys. Res., 112, D13303, doi:10.1029/2006JD008340.

Babich, L. P., R. I. Il'kaev, I. M. Kutsyk, K. I. Bakhov, and R. A. RousselDupré (2004), Self-consistent calculation of upward atmospheric discharge developing in the mode relativistic runaway electron avalanches, Geomagn. Aeron., 44(2), 231-242.

Babich, L. P., A. Y. Kudryavtsev, M. L. Kudryavtseva, and I. M. Kutsyk (2007), Terrestrial gamma-ray flashes and neutron pulses from direct simulation of gigantic upward atmospheric discharge, JETP Lett., 85 (10), 483-487, doi:10.1134/S0021364007100037.

Babich, L. P., A. Y. Kudryavtsev, M. L. Kudryavtseva, and I. M. Kutsyk (2008), Atmospheric gamma-ray and neutron flashes, J. Exp. Theor. Phys., 106, 65-76, doi:10.1007/s11447-008-1005-4.

Berman, B. L., and S. C. Fultz (1975), Measurements of the giant dipole resonance with monoenergetic photons, Rev. Mod. Phys., 47, 713-761, doi:10.1103/RevModPhys.47.713.

Bratolyubova-Tsulukidze, L., E. Grachev, O. Grigoryan, V. Kunitsyn, B. Kuzhevskij, D. Lysakov, O. Nechaev, and M. Usanova (2004), Thunderstorms as the probable reason of high background neutron fluxes at $L<1.2$, Adv. Space Res., 34(8), 1815-1818.

Carlson, B. E., N. G. Lehtinen, and U. S. Inan (2007), Constraints on terrestrial gamma ray flash production from satellite observation, Geophys. Res. Lett., 34, L08809, doi:10.1029/2006GL029229.

Carlson, B. E., N. G. Lehtinen, and U. S. Inan (2009), Terrestrial gamma ray flash production by lightning current pulses, J. Geophys. Res., 114 A00E08, doi:10.1029/2009JA014531.

Chadwick, M., et al. (2000), Handbook of photonuclear data for applications: Cross-sections and spectra, IAEA-TECDOC-1178, Int. At. Energy Agency, Vienna.

Cohen, M. B., U. S. Inan, and G. Fishman (2006), Terrestrial gamma ray flashes observed aboard the Compton Gamma Ray Observatory/Burst and Transient Source Experiment and ELF/VLF radio atmospherics, J. Geophys. Res., 111, D24109, doi:10.1029/2005JD006987.

Cullen, D., J. Hubbell, and L. Kissel (1997), EPDL97: The evaluated photo data library97 version, Tech. Rep. UCRL-50400-Vol. 6-Rev. 5, Lawrence Livermore Natl. Lab., Livermore, Calif.

Cummer, S. A., Y. Zhai, W. Hu, D. M. Smith, L. I. Lopez, and M. A. Stanley (2005), Measurements and implications of the relationship between lightning and terrestrial gamma ray flashes, Geophys. Res. Lett., 32, L08811, doi:10.1029/2005GL022778.

Dwyer, J. R. (2005), The initiation of lightning by runaway air breakdown, Geophys. Res. Lett., 32, L20808, doi:10.1029/2005GL023975.

Dwyer, J. R. (2008), Source mechanisms of terrestrial gamma-ray flashes, J. Geophys. Res., 113, D10103, doi:10.1029/2007JD009248.

Dwyer, J. R., and D. M. Smith (2005), A comparison between Monte Carlo simulations of runaway breakdown and terrestrial gamma-ray flash observations, Geophys. Res. Lett., 32, L22804, doi:10.1029/2005GL023848.

Dwyer, J. R., et al. (2004), A ground level gamma-ray burst observed in association with rocket-triggered lightning, Geophys. Res. Lett., 31, L05119, doi:10.1029/2003GL018771.

Fargion, D. (2002), Discovering ultra-high-energy neutrinos through horizontal and upward $\tau$ air showers: Evidence in terrestrial gamma flashes?, Astrophys. J., 570, 909-925, doi:10.1086/339772.
Fishman, G. J., et al. (1994), Discovery of intense gamma-ray flashes of atmospheric origin, Science, 264(5163), 1313-1316.

Grefenstette, B. W., D. M. Smith, J. R. Dwyer, and G. J. Fishman (2008), Time evolution of terrestrial gamma ray flashes, Geophys. Res. Lett., 35, L06802, doi:10.1029/2007GL032922.

Grefenstette, B. W., D. M. Smith, B. J. Hazelton, and L. I. Lopez (2009), First RHESSI terrestrial gamma ray flash catalog, J. Geophys. Res., 114, A02314, doi:10.1029/2008JA013721.

Gurevich, A. V., K. P. Zybin, and R. A. Roussel-Dupré (1999), Lightning initiation by simultaneous effect of runaway breakdown and cosmic ray showers, Phys. Lett. A, 254(1-2), 79-87, doi:10.1016/S0375-9601(99) 00091-2.

Hedin, A. E. (1991), Extension of the MSIS thermosphere model into the middle and lower atmosphere, J. Geophys. Res., 96, 1159-1172, doi:10.1029/90JA02125.

Inan, U. S., and N. G. Lehtinen (2005), Production of terrestrial gamma-ray flashes by an electromagnetic pulse from a lightning return stroke, Geophys. Res. Lett., 32, L19818, doi:10.1029/2005GL023702.

Inan, U. S., M. B. Cohen, R. Said, D. M. Smith, and L. I. Lopez (2006), Terrestrial gamma-ray flashes and lightning discharges, Geophys. Res. Lett., 33, L18802, doi:10.1029/2006GL027085.

Kossov, M. V. (2002), Approximation of photonuclear interaction crosssections, Eur. Phys. J. A, 14, 377-392.

Lehtinen, N. G., M. Walt, U. S. Inan, T. F. Bell, and V. P. Pasko (1996), $\gamma$-ray emission produced by a relativistic beam of runaway electrons accelerated by quasi-electrostatic thundercloud fields, Geophys. Res. Lett., 23(19), 2645-2648, doi:10.1029/96GL02573.

Lehtinen, N. G., T. F. Bell, and U. S. Inan (1999), Monte Carlo simulation of runaway $\mathrm{MeV}$ electron breakdown with application to red sprites and terrestrial gamma ray flashes, J. Geophys. Res., 104(A11), 24,69924,712, doi:10.1029/1999JA900335.

Libby, L., and H. Lukens (1973), Production of radiocarbon in tree rings by lightning bolts, J. Geophys. Res., 78, 5902-5903.

Marshall, T. C., M. Stolzenburg, C. R. Maggio, L. M. Coleman, P. R. Krehbiel, T. Hamlin, R. J. Thomas, and W. Rison (2005), Observed electric fields associated with lightning initiation, Geophys. Res. Lett., 32, L03813, doi:10.1029/2004GL021802.

Milikh, G., and J. A. Valdivia (1999), Model of gamma ray flashes due to fractal lightning, Geophys. Res. Lett., 26(4), 525-528, doi:10.1029/ 1999GL900001.

Milikh, G. M., P. N. Guzdar, and A. S. Sharma (2005), Gamma ray flashes due to plasma processes in the atmosphere: Role of whistler waves, J. Geophys. Res., 110, A02308, doi:10.1029/2004JA010681.

Paiva, G. S., A. C. Pavão, and C. C. Bastos (2009), "Seed" electrons from muon decay for runaway mechanism in the terrestrial gamma ray flash production, J. Geophys. Res., 114, D03205, doi:10.1029/2008JD010468. Roussel-Dupré, R. A., and A. V. Gurevich (1996), On runaway breakdown and upward propagating discharges, J. Geophys. Res., 101(A2), $2297-$ 2312, doi:10.1029/95JA03278

Shah, G. N., H. Razdan, Q. M. Ali, and C. L. Bhat (1985), Neutron generation in lightning bolts, Nature, 313, 773-775, doi:10.1038/313773a0.

Shyam, A., and T. C. Kaushik (1999), Observation of neutron bursts associated with atmospheric lightning discharge, J. Geophys. Res., 104, 6867-6870, doi:10.1029/98JA02683.

Smith, D. M., L. I. Lopez, R. P. Lin, and C. P. Barrington-Leigh (2005), Terrestrial gamma flashes observed up to $20 \mathrm{MeV}$, Science, 307(5712), 1085-1088, doi:10.1126/science.1107466.

Stanley, M. A., X.-M. Shao, D. M. Smith, L. I. Lopez, M. B. Pongratz, J. D. Harlin, M. Stock, and A. Regan (2006), A link between terrestrial gamma-ray flashes and intracloud lightning discharges, Geophys. Res. Lett., 33, L06803, doi:10.1029/2005GL025537.

Tierney, H. E., R. A. Roussel-Dupré, E. M. D. Symbalisty, and W. H. Beasley (2005), Radio frequency emissions from a runaway electron avalanche model compared with intense, transient signals from thunderstorms, J. Geophys. Res., 110, D12109, doi:10.1029/2004JD005381.

B. E. Carlson and N. G. Lehtinen, STAR Lab, Electrical Engineering Department, Stanford University, 350 Serra Mall, Packard Bldg., Stanford, CA 94305-9515, USA. (brantc@stanford.edu)

U. S. Inan, Koç University, Rumelifeneri Yolu, 34450 Sariyer, Istanbul, Turkey. 